\title{
UV OH spectrum used as a molecular pyrometer
}

\section{Charles de Izarra}

Faculté des Sciences, Site de Bourges, Université d'Orléans, rue Gaston Berger, BP 4043, 18028 Bourges Cedex, France

Received 19 April 2000

\begin{abstract}
For the UV OH band at $306.357 \mathrm{~nm}$ (transition $\mathrm{A}^{2} \Sigma, v=0 \rightarrow \mathrm{X}^{2} \Pi, v^{\prime}=0$ ) that is frequently observed in hot gases containing oxygen and hydrogen (flames, arc plasmas), a very sensitive variation of two groups of unresolved rotational lines as a function of the temperature has been found. Using a numerical simulation, this variation has been calibrated as a function of the temperature and of the optical apparatus function. This calibration allows us to easily determine the rotational temperature without computing any line intensity.
\end{abstract}

\section{Introduction}

Molecular spectra [1] can successfully give information in the temperature range $2000-8000 \mathrm{~K}$ where atomic spectra are not strong enough to ensure a good sensitivity [2]. To determine the rotational temperature of a diatomic gas, that is generally close to its heavy species kinetic temperature, a possible method is the well known Boltzmann plot, assuming a rather high resolution optical device is available to resolve rotational lines. On the other hand - and it is most frequently the case with in situ industrial studies-molecular spectra are recorded with a poor spectral resolution that does not allow the observation of the resolved rotational lines. In this case, it is useful to compare experimental data with a computed synthetic spectrum that takes into account both the temperature and the optical apparatus function effects [3]. However, the comparison of experimental data with a synthetic molecular spectrum is often difficult to perform and previous published works do not give precise details of the fitting procedure employed. In addition, to determine the temperature, most authors deal with intensities of unresolved groups of rotational lines supposing the definition of a given spectral range. In this work, from a numerical simulation of the UV OH spectrum and an original normalization of the synthetic spectra, it has been possible to produce a calibration of the maximum amplitude of two groups of unresolved rotational lines, which are very sensitive, as a function of the temperature.

\section{The UV OH spectrum}

\subsection{Generalities}

The intensity $I_{n m}$ of a spectral line corresponding to a transition $(n \rightarrow m)$ between two levels is given by

$$
I_{n m}=N_{n} A_{n m} h v_{n m}
$$

where $h$ is Planck's constant, $v_{n m}$ the frequency of the transition, $A_{n m}$ the spontaneous transition probability and $N_{n}$ the particle density in the initial state $|n\rangle$. A simple thermodynamics model commonly employed, giving $N_{n}$ as a function of the temperature $T$, may be found, assuming thermodynamics equilibrium and Boltzmann's law

$$
N_{n}=\frac{N_{0} g_{n}}{Z(T)} \exp \left(-\frac{E_{n}}{k T}\right)
$$

where $k$ is the Boltzmann constant, $N_{0}$ the particle density, $Z(T)$ the partition function of the particle, $g_{n}$ and $E_{n}$ the statistical weight and the energy of the state $|n\rangle$, respectively. From equations (1) and (2), another expression of $I_{n m}$ is

$$
I_{n m}=\frac{K_{n m}}{Z(T)} \exp \left(-\frac{E_{n}}{k T}\right)
$$

where $K_{n m}=N_{0} A_{n m} g_{n} h v_{n m}$ is a constant for a given transition $(n \rightarrow m)$.

In the case when $I_{n m}$ is known for a given temperature $T_{\text {ref }}\left(I_{n m_{\text {ref }}}\right.$ is taken for reference), we can write:

$$
I_{n m}=I_{n m_{\mathrm{ref}}} \frac{Z\left(T_{\mathrm{ref}}\right)}{Z(T)} \exp \left(-\frac{E_{n}\left(T_{\mathrm{ref}}-T\right)}{T_{\mathrm{ref}} T}\right) .
$$

At least, it is important to emphasize that the general procedure of temperature measurements from molecular band spectra does not need to consider absolute values of light intensities. Since, most of the time, experimental data are relative values, we can consider only theoretical relative values, as given in equation (4).

\subsection{The UV OH spectrum}

The OH UV spectrum is frequently observed in many kinds of flames and hot gases containing oxygen and hydrogen. In addition, the rotational temperature of the $\mathrm{OH}$ radical is close to the gas temperature [3], i.e. to the temperature 


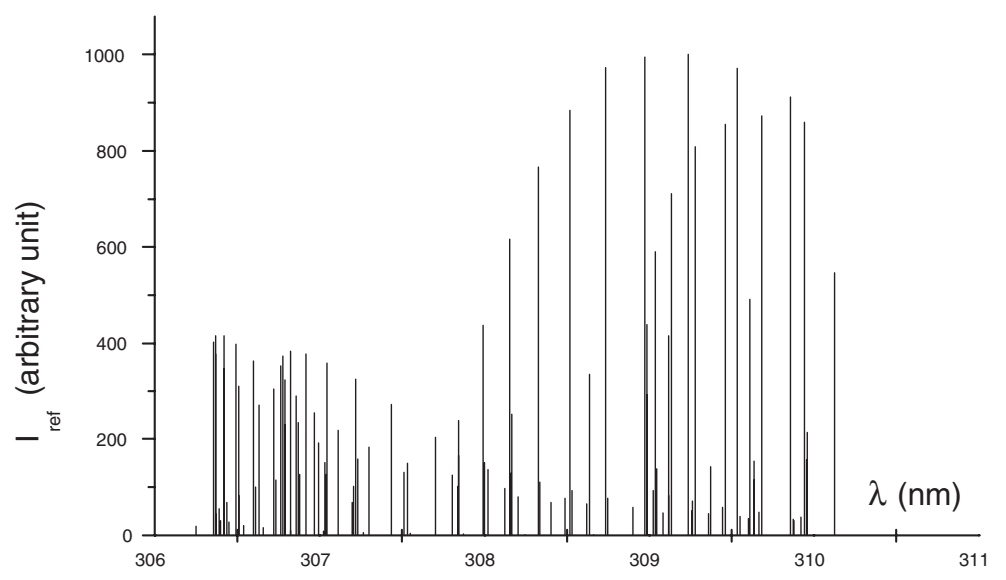

Figure 1. Plot of the rotational lines of $\mathrm{OH}$ spectrum represented as Dirac impulses for the reference temperature $T_{\text {ref }}=3000 \mathrm{~K}$. Reference data are from the paper by Diecke and Crosswhite [4].

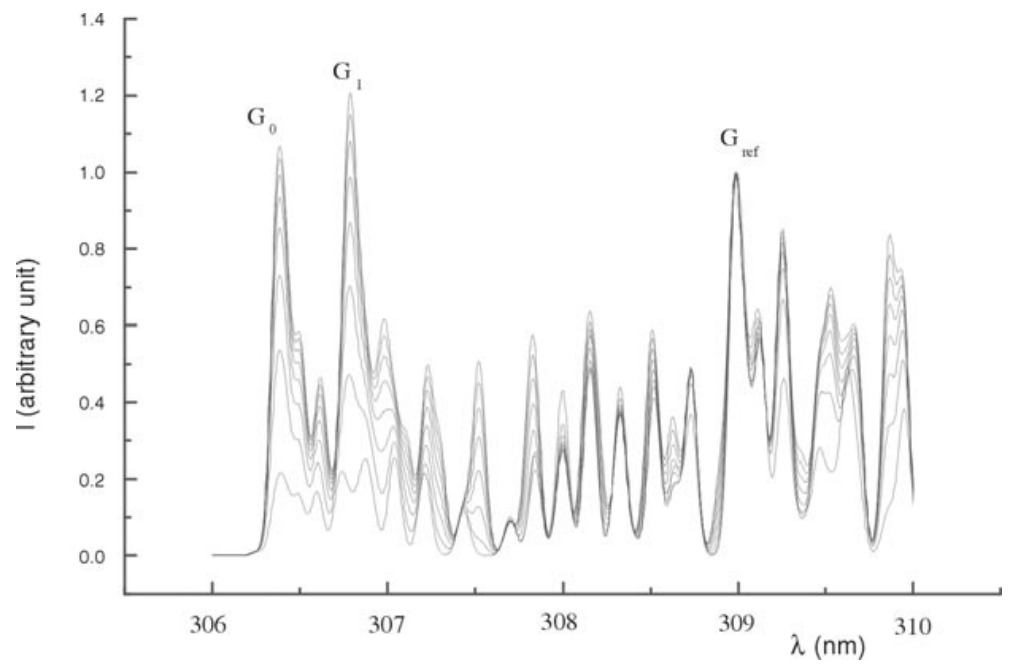

Figure 2. Plot of the UV OH spectrum for the rotational temperature varying from $1000-8000 \mathrm{~K}$, in $1000 \mathrm{~K}$ steps $(\Delta=0.1 \mathrm{~nm})$.

determined using an enthalpy balance. The $\mathrm{OH}$ band $\left(\mathrm{A}^{2} \Sigma, v=0 \rightarrow \mathrm{X}^{2} \Pi, v^{\prime}=0\right)$ has been studied thoroughly by Dieke and Crosswhite [4], and shows a red degradation with four main band heads $\mathrm{R}_{1}, \mathrm{R}_{2}, \mathrm{Q}_{1}, \mathrm{Q}_{2}$ at $306.537 \mathrm{~nm}$, $306.776 \mathrm{~nm}, 307.844 \mathrm{~nm}$ and $308.986 \mathrm{~nm}$, respectively. The paper by Diecke and Crosswhite [4] gives the wavelengths of the different rotational lines of the different branches, the energy of the upper level of each transition (in $\mathrm{cm}^{-1}$ ), and the normalized intensity $I_{n m_{\text {ref }}}$ of each line for a reference temperature $T_{\text {ref }}=3000 \mathrm{~K}$.

Figure 1 gives a plot of the rotational lines represented as Dirac impulses for the reference temperature $T_{\text {ref }}=$ $3000 \mathrm{~K}$. Rotational lines are presented in table 1 , and the corresponding data are used as input data in the simulation program. We have to emphasize that some weak transitions are given in [4] without being attributed to any coherent transition of the $\mathrm{OH}$ spectrum. Consequently, these weak transitions have been omitted in our procedure.

\subsection{Optical apparatus function}

In experimental spectroscopy, it is necessary to measure the apparatus function either by employing a laser line (that may be considered as a Dirac impulse $\left.\delta\left(\lambda_{0}\right)\right)$ or by employing sharp lines emitted by a spectral lamp (a low pressure mercury lamp for example). In many experimental conditions, the apparatus function (i.e. the impulse response of the optical device) can be well fitted with a Gaussian profile as a function of the wavelength $\lambda$. In addition, lines emitted by hot gases are broadened by different effects [3]. Consequently, the best way to mathematically represent the apparatus function is to consider a normalized Gaussian profile given by:

$$
G(\lambda)=\frac{2}{\Delta \sqrt{\pi}} \exp \left(-\frac{\left(\lambda-\lambda_{0}\right)^{2}}{(\Delta / 2)^{2}}\right)
$$

where $\Delta$ represents the full width at $1 / \mathrm{e}$ of the maximum located at the wavelength $\lambda_{0}$.

\section{Numerical simulation: calibration}

Using reference data and equation (4), a Dirac synthetic spectrum is first computed for a given temperature $T$, assuming that $Z\left(T_{\text {ref }}\right) / Z(T)=1$ in equation (4). Then, the Dirac spectrum is convoluted with a Gaussian apparatus function (equation (5)) to produce a synthetic spectrum. 
UV OH spectrum used as a molecular pyrometer

Table 1. Parameters of the rotational lines used to proceed to numerical simulation of the UV OH molecular band. Reference data are from the paper by Diecke and Crosswhite [4].

\begin{tabular}{|c|c|c|c|c|c|c|c|c|}
\hline $\begin{array}{l}\lambda \\
(\mathrm{nm})\end{array}$ & $\begin{array}{l}I_{\text {ref }} \\
(3000 \mathrm{~K})\end{array}$ & $\begin{array}{l}E_{u} \\
\left(\mathrm{~cm}^{-1}\right)\end{array}$ & $\begin{array}{l}\lambda \\
(\mathrm{nm})\end{array}$ & $\begin{array}{l}I_{\text {ref }} \\
(3000 \mathrm{~K})\end{array}$ & $\begin{array}{l}E_{u} \\
\left(\mathrm{~cm}^{-1}\right)\end{array}$ & $\begin{array}{l}\lambda \\
(\mathrm{nm})\end{array}$ & $\begin{array}{l}I_{\text {ref }} \\
(3000 \mathrm{~K})\end{array}$ & $\begin{array}{l}E_{u} \\
\left(\mathrm{~cm}^{-1}\right)\end{array}$ \\
\hline 306.2523 & 19 & 32643.45 & 307.2009 & 69 & 32542.56 & 308.9861 & 62 & 33383.26 \\
\hline 306.3565 & 402 & 34282.99 & 307.2063 & 102 & 32541.98 & 308.9861 & 293 & 32541.98 \\
\hline 306.3725 & 415 & 33951.80 & 307.2199 & 325 & 33148.73 & 308.9861 & 95 & 32542.56 \\
\hline 306.3725 & 378 & 34645.53 & 307.2308 & 159 & 37443.91 & 308.9861 & 439 & 32643.45 \\
\hline 306.3725 & 44 & 34280.64 & 307.2660 & 6 & 37440.15 & 308.9861 & 100 & 32644.22 \\
\hline 306.3921 & 55 & 33949.67 & 307.3028 & 183 & 36902.90 & 309.0270 & 94 & 32779.49 \\
\hline 306.3970 & 31 & 34642.92 & 307.4369 & 273 & 32947.05 & 309.0364 & 589 & 32778.49 \\
\hline 306.4189 & 415 & 33652.29 & 307.5123 & 131 & 38007.90 & 309.0449 & 70 & 32474.62 \\
\hline 306.4236 & 346 & 35038.61 & 307.5334 & 151 & 37440.15 & 309.0473 & 139 & 32474.30 \\
\hline 306.4370 & 68 & 33650.38 & 307.5486 & 5 & 38003.93 & 309.0862 & 47 & 40513.79 \\
\hline 306.4491 & 27 & 35035.86 & 307.7028 & 204 & 32778.49 & 309.1186 & 416 & 32542.56 \\
\hline 306.4950 & 397 & 33384.97 & 307.8071 & 125 & 38003.93 & 309.1186 & 83 & 32948.31 \\
\hline 306.5095 & 310 & 35462.01 & 307.8373 & 102 & 38597.79 & 309.1361 & 712 & 32947.05 \\
\hline 306.5095 & 83 & 33383.26 & 307.8440 & 239 & 32474.62 & 309.2394 & 1000 & 33652.29 \\
\hline 306.5372 & 20 & 35459.02 & 307.8468 & 166 & 32474.30 & 309.2577 & 50 & 33650.38 \\
\hline 306.5976 & 363 & 33150.14 & 307.8753 & 3 & 38003.93 & 309.2650 & 71 & 33148.73 \\
\hline 306.6114 & 100 & 33148.73 & 307.9951 & 437 & 32542.56 & 309.2786 & 808 & 33148.73 \\
\hline 306.6318 & 271 & 35914.82 & 308.0006 & 152 & 32541.98 & 309.3609 & 45 & 40513.79 \\
\hline 306.6613 & 15 & 35911.59 & 308.0231 & 138 & 32643.45 & 309.3722 & 143 & 32440.61 \\
\hline 306.7240 & 304 & 32948.31 & 308.1255 & 98 & 38593.62 & 309.4459 & 58 & 33150.14 \\
\hline 306.7356 & 114 & 32947.05 & 308.1541 & 616 & 32644.22 & 309.4618 & 855 & 33383.26 \\
\hline 306.7661 & 352 & 34642.92 & 308.1620 & 130 & 32643.45 & 309.5342 & 973 & 33951.80 \\
\hline 306.7775 & 373 & 34280.64 & 308.1665 & 252 & 32440.61 & 309.5546 & 40 & 33949.67 \\
\hline 306.7929 & 230 & 36396.66 & 308.2065 & 80 & 39212.69 & 309.6000 & 35 & 41198.19 \\
\hline 306.7929 & 323 & 35035.86 & 308.2456 & 2 & 39208.99 & 309.6124 & 492 & 32644.22 \\
\hline 306.8277 & 11 & 36393.24 & 308.3278 & 766 & 32779.49 & 309.6349 & 114 & 32474.30 \\
\hline 306.8277 & 383 & 33949.67 & 308.3374 & 111 & 32778.49 & 309.6349 & 154 & 32474.62 \\
\hline 306.8608 & 290 & 35459.02 & 308.4050 & 68 & 32541.98 & 309.6650 & 48 & 33384.97 \\
\hline 306.8704 & 234 & 32779.49 & 308.4894 & 77 & 39208.99 & 309.6830 & 873 & 33650.38 \\
\hline 306.8799 & 126 & 32778.49 & 308.5196 & 884 & 32948.31 & 309.8586 & 912 & 34282.99 \\
\hline 306.9177 & 378 & 33650.38 & 308.5317 & 93 & 32947.05 & 309.8715 & 34 & 41193.51 \\
\hline 306.9675 & 255 & 35911.59 & 308.6226 & 66 & 39851.66 & 309.8807 & 31 & 34280.64 \\
\hline 306.9913 & 193 & 36906.50 & 308.6390 & 335 & 32474.62 & 309.9210 & 38 & 33949.67 \\
\hline 307.0244 & 9 & 36902.90 & 308.6634 & 2 & 39847.20 & 309.9411 & 860 & 33949.67 \\
\hline 307.0318 & 152 & 32644.22 & 308.7338 & 974 & 33150.14 & 309.9538 & 158 & 32644.22 \\
\hline 307.0392 & 127 & 32643.45 & 308.7481 & 77 & 33148.73 & 309.9593 & 215 & 32541.98 \\
\hline 307.0478 & 359 & 33383.26 & 308.9008 & 59 & 39847.20 & 309.9593 & 33 & 32440.61 \\
\hline 307.1145 & 218 & 36393.24 & 308.9734 & 995 & 33384.97 & & & \\
\hline
\end{tabular}

A set of about 2000 synthetic spectra has been computed for temperatures varying from $600-9000 \mathrm{~K}$, in $200 \mathrm{~K}$ steps, and for $\Delta$ varying from $0.02-0.98 \mathrm{~nm}$, in $0.02 \mathrm{~nm}$ steps. This data set is stored on disc for data processing.

Figure 2 gives a plot of synthetic spectra for an apparatus function width $\Delta=0.1 \mathrm{~nm}$ and for a rotational temperature varying from $1000-8000 \mathrm{~K}$, in $1000 \mathrm{~K}$ steps. An important point is that all the synthetic spectra have been normalized against the intensity of the group of unresolved rotational lines $G_{\text {ref }}$ (see figure 2 ) at $\approx 309 \mathrm{~nm}$, which appears to be the relative strongest group of unresolved lines when the rotational temperature is lower than $4000 \mathrm{~K}$.

A quick survey of figure 2 shows the presence of two groups of unresolved rotational lines, $G_{0}$ and $G_{1}$, the amplitudes of which are very sensitive against the rotational temperature. A more detailed analysis of the groups $G_{0}$ and $G_{1}$ shows that the wavelength of their maximum amplitude varies as a function of the temperature $T$.

In order to avoid the difficulties encountered in experimental measurements of group line intensities it has been decided to only consider the maximum amplitude of the groups of lines $G_{0}$ and $G_{1}$ as a function of the temperature.
It is obvious that this choice is dependent on the apparatus function width $\Delta$ that must be precisely determined.

The typical evolution of the maximum amplitude of $G_{0} / G_{\text {ref }}$ and $G_{1} / G_{\text {ref }}$ as a function of the rotational temperature is given in figure 3 for an apparatus function width $\Delta=0.12 \mathrm{~nm}$. From figure 3 , we notice a high sensitivity of these maxima in the temperature range 1000 $4000 \mathrm{~K}$. This sensitivity decreases when the temperature is higher than $4000 \mathrm{~K}$, and then leads to an increase of the error percentage in a temperature determination.

For a practical determination of the temperature from an experimental $\mathrm{UV} \mathrm{OH}$ spectrum recorded with a known apparatus function width, we give the tables 2, 3, 4 and 5 that present the ratios $G_{0} / G_{\text {ref }}$ and $G_{1} / G_{\text {ref }}$ for different values of temperature $T$ and apparatus function width $\Delta$.

\section{Check of the method}

\subsection{Published works}

A check of the temperature determination method has been made by considering the $\mathrm{OH}$ spectra given in [3]. The authors 
C de Izarra

Table 2. Maximum amplitude of the unresolved group of rotational lines $G_{0}$ as a function of the temperature and for $\Delta$ varying from $0.04-0.2 \mathrm{~nm}$.

\begin{tabular}{|c|c|c|c|c|c|c|c|c|c|}
\hline$T(\mathrm{~K})$ & $0.04 \mathrm{~nm}$ & $0.06 \mathrm{~nm}$ & $0.08 \mathrm{~nm}$ & $0.10 \mathrm{~nm}$ & $0.12 \mathrm{~nm}$ & $0.14 \mathrm{~nm}$ & $0.16 \mathrm{~nm}$ & $0.18 \mathrm{~nm}$ & $0.2 \mathrm{~nm}$ \\
\hline 600 & 04633 & .05345 & 7 & 0.06899 & 0.07 & 0.074 & & 0.080 & 0 \\
\hline 800 & & & & & & & & & \\
\hline 1000 & & & & & & & & & \\
\hline 1200 & 0 & 0.25 & 6 & 0.29 & 0.29 & 0.30 & 5 & & 60 \\
\hline 1400 & 6 & 76 & 2 & 0.362 & 0 & 0. & & & 551 \\
\hline 1600 & 49 & 35 & 65 & 0.42 & & & & & \\
\hline 1800 & 40517 & 0.42798 & 0.46083 & 0.484 & 0.496 & 0.50612 & & & \\
\hline 2000 & .44964 & 0.47415 & 0.51035 & 0.53653 & 0.550 & 0.56064 & 0.568 & 0.571 & 0.56942 \\
\hline 200 & 0.48947 & 0.51548 & 0.55482 & 0.58355 & 0.59876 & 0.60978 & 0.617 & 0.620 & 0.61906 \\
\hline 100 & 0.52523 & 0.55256 & 0.59482 & 0.62602 & 0.642 & 0.65407 & 0.662 & 0.66582 & 0.66360 \\
\hline 500 & 0.55742 & 0.58594 & 0.63092 & 0.66441 & 0.68183 & 0.69403 & & 0.70614 & 0.70356 \\
\hline 00 & .58652 & 0.61609 & 0.66359 & 0.69919 & & & & & 0.73942 \\
\hline 00 & & & & & & & & & 0.77160 \\
\hline 00 & & & & & & & & & \\
\hline 00 & & & & & & & & & \\
\hline 600 & & & & & & & & & \\
\hline 3800 & & & & & & & & & \\
\hline 4000 & & & & & & & & & \\
\hline 4200 & & & & & & & & & \\
\hline 4400 & & & & & & & & & \\
\hline 600 & 0 & 0. & & & & & & & \\
\hline 4800 & .76508 & 0.80734 & 0.86971 & 0.91 & & & & & 0.94781 \\
\hline 5000 & 0.77642 & 0.81959 & 0.88292 & 0.93368 & 0.95 & 0.96 & 35 & 0.967 & 0.95903 \\
\hline 5200 & 0.78703 & 0.83107 & 0.89528 & 0.94676 & 0.96 & 0.97798 & 99 & 0.97820 & 0.96917 \\
\hline 5400 & 0.79698 & 0.84183 & 0.90687 & 0.95901 & 0.98061 & 0.98946 & & 0.988 & 0.97834 \\
\hline 5600 & 0.80632 & 0.85194 & 0.91777 & 0.97050 & 192 & 1.00011 & 56 & 0.99697 & 8665 \\
\hline 5800 & 0.81512 & 0.86146 & 0.92803 & 0.98130 & 1.00249 & 1.01002 & 1.011 & 1.00514 & 0.99417 \\
\hline 5000 & 0.82341 & 0.87044 & 0.93770 & 0.99146 & 1.01 & 1.01 & 1.02 & 1.01 & 1.00100 \\
\hline 6200 & 0.83124 & 0.87892 & 0.94683 & 1.00105 & 1.02172 & 1.02785 & 783 & $1.01 \mathrm{c}$ & 1.00720 \\
\hline 6400 & 0.83865 & 0.88 & 0.95547 & 1.01010 & 1.03048 & 1.0 & & 73 & 1.01284 \\
\hline 6600 & 0.84566 & 0.89 & 5366 & 1.01866 & & & & & 796 \\
\hline 6800 & 0.85231 & 0.90176 & & & & & & & 1.02261 \\
\hline 7000 & & & & 1.03445 & 1.05383 & & & 1.04160 & 1.02685 \\
\hline 7200 & 0.86464 & & & & & & & & 1.03071 \\
\hline 7400 & 0.87036 & & & & & & & & \\
\hline 7600 & & & & & & & & & 1.03743 \\
\hline & & & & & & & & & \\
\hline 8000 & 0.88599 & 0.93792 & 1.01072 & 1.06757 & 1.08508 & 1.08462 & 1.07716 & 1.06067 & 1.04301 \\
\hline
\end{tabular}

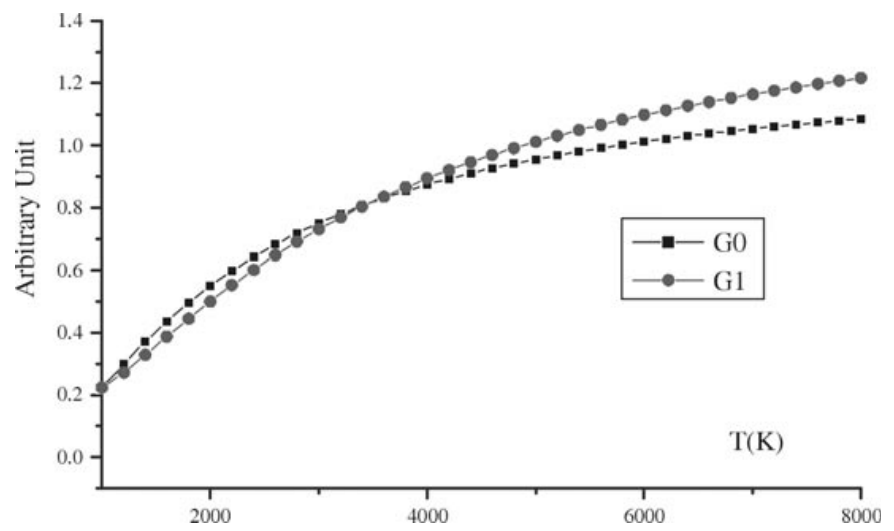

Figure 3. Plot of the maxima amplitudes of the groups of unresolved lines as a function of the temperature for the apparatus function width $\Delta=0.12 \mathrm{~nm}$.

give the apparatus function by considering the full width at half maximum (FWHM) $\delta$ of a Gaussian profile. For comparison, the $\delta$ parameter has been transformed into the full width at $1 / \mathrm{e}$ of the maximum with the relation $\delta=$ $\Delta \sqrt{\ln 2}$. In all cases, since the spectra have been recorded in plasmas with copper electrodes, only the group of unresolved lines $G_{1}$ has been considered, because of the presence of a copper line at the wavelength $\lambda=306.341 \mathrm{~nm}$ that can perturbate the amplitude of the group $G_{0}$. Pellerin et al [3] presented a figure of the $\mathrm{UV} \mathrm{OH}$ spectrum recorded on a plasma jet containing water. The spectrum was obtained with an optical apparatus function width $\Delta=0.0456 \mathrm{~nm}$. Using a rule, it is easy to measure the amplitudes of the unresolved groups of rotational lines $G_{1}$ and $G_{\text {ref }}$. Graphical 
UV OH spectrum used as a molecular pyrometer

Table 3. Maximum amplitude of the unresolved group of rotational lines $G_{0}$ as a function of the temperature and for $\Delta$ varying from $0.22-0.38 \mathrm{~nm}$.

\begin{tabular}{|c|c|c|c|c|c|c|c|c|c|}
\hline$T(\mathrm{~K})$ & $0.22 \mathrm{~nm}$ & $0.24 \mathrm{~nm}$ & $0.26 \mathrm{~nm}$ & $0.28 \mathrm{~nm}$ & $0.30 \mathrm{~nm}$ & $0.32 \mathrm{~nm}$ & $0.34 \mathrm{~nm}$ & $0.36 \mathrm{~nm}$ & $0.38 \mathrm{~nm}$ \\
\hline 600 & 0.08424 & 08660 & 0.08912 & 0.09175 & 0.09446 & 20 & 50 & & 0.10602 \\
\hline 800 & & & & & & & & & \\
\hline 1000 & & & & & & & & & \\
\hline 1200 & 31309 & 0.312 & 0.311 & 0.311 & 0.31 & 0.30 & 8 & 0.30 & 53 \\
\hline 1400 & 38491 & 5 & 3 & 88 & 32 & & & & \\
\hline 1600 & 45104 & 55 & 0.44 & 3 & 90 & & & & \\
\hline 1800 & 0.51126 & 0.50719 & 0.50250 & 0.497 & 0.49194 & 0.486 & & 0.47612 & .47235 \\
\hline 2000 & 0.56568 & 0.56069 & 0.55489 & 0.54844 & 0.54164 & 0.534 & 0.52723 & 0.52160 & 0.51674 \\
\hline 2200 & 0.61465 & 0.60889 & 0.60208 & 0.59444 & 0.58635 & 0.57827 & 0.56918 & 0.56 & 0.55662 \\
\hline 2400 & 0.65868 & 0.65220 & 0.64448 & 0.63576 & 0.62648 & 0.61721 & 0.60677 & 0.59 & 0.59229 \\
\hline 2600 & 0.69818 & 0.69105 & 0.68249 & 0.67278 & 0.66245 & 0.65209 & 0.64043 & 0.63176 & 0.62423 \\
\hline 2800 & 0.73360 & 0.72586 & 0.71 & 0.70 & & & & & 5276 \\
\hline 00 & & 0.75701 & 0.74 & & & & & & 7823 \\
\hline 200 & & & & & & & & & 098 \\
\hline 3400 & & & & & & & & & \\
\hline 600 & & & & & & & & & \\
\hline 3800 & & & & & & & & & \\
\hline 4000 & & & & & & & & & \\
\hline 200 & & & & & & & & & 98 \\
\hline 400 & 0.911 & & & & & & & & 453 \\
\hline 600 & 92515 & 0.91 & & & & & & & 486 \\
\hline 800 & 0.93704 & 0.92417 & 0.90967 & 0.89374 & 0.87684 & 0.8 & 0.84085 & 0.8 & 0.81410 \\
\hline 5000 & 0.94773 & 0.93443 & 0.91957 & 0.90333 & 0.88614 & 0.86901 & 0.84957 & 0.83 & 0.82237 \\
\hline 5200 & 0.95734 & 0.94363 & 0.92843 & 0.91191 & 0.89446 & 0.87708 & 0.85738 & 0.84 & 0.82978 \\
\hline 5400 & 0.96600 & 0.95189 & 0.93636 & 0.91957 & 0.90189 & 0.884 & 0.86436 & 0.8 & 0.83641 \\
\hline 5600 & 0.97380 & 0.95930 & 0.94346 & 0.92643 & 0.90854 & 0.89077 & 0.87062 & 0.8 & 0.84235 \\
\hline 5800 & 0.98084 & 0.96595 & 0.94982 & 0.93256 & 0.91449 & 0.89655 & 0.87622 & 0.86 & 0.84768 \\
\hline 6000 & 0.98718 & 0.97192 & 0.95552 & 0.93804 & 0.91982 & 0.90173 & 0.88124 & 0.86 & 0.85245 \\
\hline 6200 & 0.99290 & 0.97728 & 0.96062 & 0.94294 & 0.92457 & 0.90636 & 574 & 0.8 & 0.85673 \\
\hline 6400 & 0.99807 & 0.98210 & 0.96518 & 0.947 & 0.9 & 050 & 76 & & 6056 \\
\hline 6600 & 1.00274 & 0.98642 & 0.96927 & & 263 & & & & \\
\hline 6800 & & & & & & & 0.89659 & 0.88091 & 0.86707 \\
\hline 7000 & & & & & & & & & 0.86982 \\
\hline 7200 & & & & & & & & & 0.87229 \\
\hline 7400 & & & & & & & & & 0.87449 \\
\hline 7600 & 1.02006 & & & & & & & & 0.87646 \\
\hline & & & & & & & & & 0.87821 \\
\hline 8000 & 1.02483 & 1.00650 & 0.98796 & 0.96904 & 0.94991 & 0.93106 & 0.90985 & 0.89389 & 0.87978 \\
\hline
\end{tabular}

measurement gives $G_{1}=30 \mathrm{~mm}$ and $G_{\text {ref }}=41 \mathrm{~mm}$, leading to a ratio (without unity) $G_{1} / G_{\text {ref }}=0.73$. With a linear interpolation in table 4 to obtain data corresponding to $\Delta=0.0456 \mathrm{~nm}$, we find a temperature value of $4000 \mathrm{~K}$. This value is in close agreement with the temperature value of $4100 \mathrm{~K}$ proposed by the authors.

\subsection{Recombining plume of an argon plasma jet}

It is now well established that dc argon plasma jets can have two kinds of flows, depending on the values of the plasma gas flow rate [5], on the current intensity and on the anode nozzle diameter. In the case of a turbulent flow, the plasma jet is short and very noisy. On the other hand, in a laminar flow, the jet is well structured with a hot and bright core followed by a recombining plume, the length of which can reach about ten centimetres. The axial temperature profile shows a rapid decrease as a function of the distance from the anode.

In the laminar recombining plume, the temperature is generally too low to employ emission atomic spectroscopy (including line intensity ratio or line broadening) and, consequently, other methods have been used in the past, such as in situ probes, or interferometry $[6,7]$.
The temperature determination presented here is realized in the plume of a laminar argon plasma jet.

4.2.1. Experimental set-up. The experimental set-up includes a commercial dc plasma torch (SAF Soudure Autogène Française trademark) operating at atmospheric pressure in ambient air, with an axial injection of pure argon controlled with flowmeters. The arc current intensity, limited at $250 \mathrm{~A}$ is supplied by an ac/dc power converter (Soudure Autogène Française trademark). The anode is in copper, and the cathode is in tungsten; the electrodes are water cooled.

The experimental conditions presented in this paper have been chosen to produce an argon laminar jet. The experimental conditions are as follows: for an anode diameter of $3 \mathrm{~mm}$, the gas flow rate is $6 \mathrm{slm}$ and the current intensity is $120 \mathrm{~A}$.

The spectroscopic acquisition chain includes a Cromex monochromator of the Ébert-Fastié type, with a focal length of $0.5 \mathrm{~m}$. It has a 1200 grooves $\mathrm{mm}^{-1}$ grating working in the first order. Photodetection is accomplished with a Princeton OMA III equipped with a matrix CCD array having 512 rows of 1024 pixels. In the first spectroscopic order, the detector covers a spectral range of $45 \mathrm{~nm}$. $\mathrm{A} \mathrm{CaF}_{2}$ lens (transparent 
Table 4. Maximum amplitude of the unresolved group of rotational lines $G_{1}$ as a function of the temperature and for $\Delta$ varying from $0.04-0.2 \mathrm{~nm}$.

\begin{tabular}{|c|c|c|c|c|c|c|c|c|c|}
\hline$T(\mathrm{~K})$ & $0.04 \mathrm{~nm}$ & $0.06 \mathrm{~nm}$ & $0.08 \mathrm{~nm}$ & $0.10 \mathrm{~nm}$ & $0.12 \mathrm{~nm}$ & $0.14 \mathrm{~nm}$ & $0.16 \mathrm{~nm}$ & $0.18 \mathrm{~nm}$ & $0.2 \mathrm{~nm}$ \\
\hline 600 & 0.07420 & 12148 & & & & 0.1401 & 66 & 0.166 & 24 \\
\hline 800 & & & & & & & & & \\
\hline 1000 & & & 0.20 & & & & & & \\
\hline 1200 & & & & & & & & & \\
\hline 1400 & & & & & & & & & \\
\hline 1600 & 0 & 0.29 & 0. & 0.36 & & & & & 6884 \\
\hline 1800 & 09 & 33 & & 0.4 & & & & & \\
\hline 2000 & 15 & 0.40 & 0.44 & 0.47 & & 0.5 & & & \\
\hline 2200 & .40542 & 0.45273 & 0.49 & 0.524 & 18 & 0.58 & 0.60 & & \\
\hline 2400 & 44899 & 0.50021 & 0.54333 & 0.57340 & 0.60220 & 0.630 & 0.65 & 18 & 0.68046 \\
\hline 00 & 49057 & 0.54477 & 0.58907 & 0.61917 & 0.648 & 0.67 & 0.6 & 97 & 83 \\
\hline 00 & 52961 & 0.58654 & 0.63190 & 0.66220 & 0.691 & 0.71 & 0.7 & 0.75 & 0.765 \\
\hline 00 & 56624 & 0.62567 & 0.67 & 0.702 & 0.731 & 0.75 & 0.7 & & 63 \\
\hline 00 & & & & & & & & & \\
\hline 00 & & & & & & & & & \\
\hline 00 & & & & & & & & & \\
\hline 300 & & & & & & & & & \\
\hline 4000 & & & & & & & & & \\
\hline 4200 & & & & & & & & & \\
\hline 4400 & & & & & & & & & \\
\hline 600 & & & & & & & & & \\
\hline 300 & 3 & & & & & & & & \\
\hline 5000 & 71 & 0.9 & & & & & & & 814 \\
\hline 5200 & .84326 & 0.92 & $0.9^{7}$ & 1.008 & & & 1.05 & 1.063 & 1.06311 \\
\hline 5400 & 0.86086 & 0.94719 & 0.99816 & 1.02749 & 1.04926 & 1.06590 & 1.07519 & 1.07775 & 1.07691 \\
\hline 5600 & 0.87758 & 0.96542 & 1.01668 & 1.04557 & 1.06658 & 1.08209 & 1.090 & 1.09134 & 1.08966 \\
\hline 5800 & 0.89347 & 0.98275 & 1.03427 & 1.06273 & 1.08295 & 1.09732 & 1.10409 & 1.10396 & 1.10143 \\
\hline 000 & 0.90859 & 0.99924 & 1.05101 & 1.07902 & 1.09 & 1.11166 & 1.117 & 1.11570 & 1.11232 \\
\hline 5200 & 0.92301 & 1.01496 & 1.06696 & 1.09451 & 1.11313 & 1.12519 & 1.12943 & 1.12662 & 1.12240 \\
\hline 6400 & 0.93675 & 1.02995 & 1.08215 & 1.10926 & 1.12707 & 1.13796 & 1.14094 & 1.13680 & 1.13174 \\
\hline 6600 & 0.94988 & 1.04426 & 1.09666 & 1.12331 & 1.14030 & 1.15003 & 1.15175 & 1.14629 & 1.14041 \\
\hline 6800 & 0.96242 & 1.05794 & 1.11051 & 1.13671 & 1.15289 & 1.16145 & 1.16192 & 1.15515 & 1.14846 \\
\hline 7000 & 0.97442 & 1.07102 & & & & & & & 1.15594 \\
\hline 7200 & & & 1.13643 & 1.16173 & 1.17627 & 1.18253 & 1.18053 & 1.17119 & 1.16290 \\
\hline 7400 & 0.99692 & & & & & & & & 1.16938 \\
\hline 7600 & 1.00748 & 1.10681 & 1.16020 & & & & & & 1.17542 \\
\hline 7800 & 1.01761 & 1.11774 & 1.17137 & 1.19534 & & 1.210 & & 1.191 & 1.18106 \\
\hline 8000 & 1.02734 & 1.12824 & 1.18209 & 1.20562 & 1.21693 & 1.21867 & 1.21191 & 1.19766 & 1.18633 \\
\hline
\end{tabular}

in the UV spectrum) forms the image of the plasma jet with a magnification of 1. A quartz optical fibre put in the focal plane of the lens is used to sample plasma radiation along a diameter of the plasma column.

We have checked that the intensity ratio of two atomic lines of $\mathrm{Cu}_{\mathrm{I}}$ did not change along different cords of the plasma column. This observation means that the temperature profile is rather flat along a diameter of the recombining plasma column. The apparatus function of the optical device has been measured using a spectral mercury lamp. The shape of a line has been successfully fitted with a Gaussian profile and leads to a full width at $1 / \mathrm{e}$ of the maximum of $0.2 \mathrm{~nm}$. Let us emphasize that the resolution of the optical system is too low to observe a separation of the rotational lines of the UV $\mathrm{OH}$ spectrum. The spectroscopic investigation is made in the near UV, in the range $290-330 \mathrm{~nm}$.

4.2.2. Results and data processing. An efficient method to observe the $\mathrm{OH}$ spectrum in a plasma jet consists of introducing a small percentage of water vapour into the plasma [3]. A first attempt has been made by bubbling the working gas (pure argon) in through water at the input of the device. From our observations, it was very difficult to control

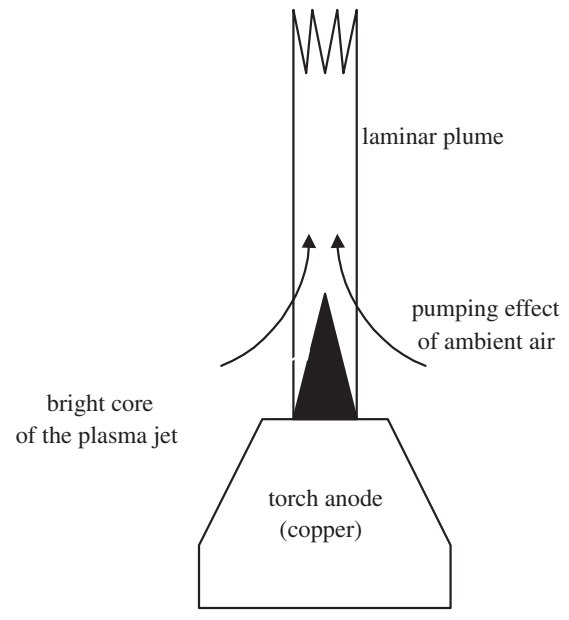

Figure 4. Pump effect of the laminar flow in ambient air.

the fraction of water injected into the plasma torch. At least, the effect of water vapour condensation on the cooled parts of the torch produced important instabilities of the plasma jet with a typical time of about $10 \mathrm{~s}$. 
UV OH spectrum used as a molecular pyrometer

Table 5. Maximum amplitude of the unresolved group of rotational lines $G_{1}$ as a function of the temperature and for $\Delta$ varying from $0.22-0.38 \mathrm{~nm}$

\begin{tabular}{|c|c|c|c|c|c|c|c|c|c|}
\hline$T(\mathrm{~K})$ & $0.22 \mathrm{~nm}$ & $0.24 \mathrm{~nm}$ & $0.26 \mathrm{~nm}$ & $0.28 \mathrm{~nm}$ & $0.30 \mathrm{~nm}$ & $0.32 \mathrm{~nm}$ & $0.34 \mathrm{~nm}$ & $0.36 \mathrm{~nm}$ & $0.38 \mathrm{~nm}$ \\
\hline 600 & 0.18275 & 18974 & 0.19661 & 0.20343 & 0.21027 & 0.21704 & 0.225 & 0.23146 & 0.23771 \\
\hline 800 & & & & & 0.264 & & & & \\
\hline 1000 & & & & & & & & & \\
\hline 1200 & 0.355 & 0.362 & 0.368 & 0.373 & 0.378 & 0.38 & 0.389 & 0.39 & 94 \\
\hline 1400 & 0.41658 & 0.42272 & 0.427 & 0.432 & 0.436 & 54 & 14 & & \\
\hline 1600 & 0.47624 & 0.48 & 0.486 & 0.489 & 0.493 & 0.49 & & & \\
\hline 1800 & 0.53356 & 0.53862 & 0.54237 & 0.54526 & 0.54757 & 0.54951 & 0.55184 & 0.55401 & 0.55653 \\
\hline 2000 & 0.58791 & 0.59245 & 0.59564 & 0.59774 & 0.59916 & 0.60021 & 0.60147 & 0.60284 & 0.60465 \\
\hline 2200 & 0.63888 & 0.64298 & 0.64566 & 0.64706 & 0.64764 & 0.64784 & 0.64810 & 0.64873 & 0.64991 \\
\hline 2400 & 0.68637 & 0.69007 & 0.69227 & 0.69304 & 0.69286 & 0.69227 & 0.69160 & 0.69155 & 0.69209 \\
\hline 2600 & 0.73039 & 0.73373 & 0.73548 & 0.73567 & 0.73481 & 0.73349 & 0.73198 & 0.73125 & 0.73125 \\
\hline 800 & 0.77103 & 0.77404 & 0.77536 & 0.77502 & 0.77355 & 0.77157 & 0.76929 & 0.76794 & 0.76742 \\
\hline 000 & 0.80845 & 0.81112 & 0.81204 & 0.811 & 0.80921 & 0.80663 & 0.80361 & 0174 & 0.80072 \\
\hline 200 & & & & & & & & & \\
\hline 400 & & & & & & & & & \\
\hline 600 & & & & & & & & & \\
\hline 3800 & 0.9 & & & & & & & & \\
\hline 4000 & & & & & & & & & \\
\hline 4200 & & & & & & & & & \\
\hline 400 & 0.99 & 0.99 & 0.99 & 0.99 & & & & & \\
\hline 600 & 1.01509 & 1.01473 & 1.01 & 1.00 & & & & & \\
\hline 800 & 1.03216 & 1.03142 & 1.02962 & 1.02616 & 1.02119 & 1.01541 & 1.00849 & 1.00358 & 0.99975 \\
\hline 5000 & 1.04783 & 1.04670 & 1.04465 & 1.04101 & 1.03585 & 1.02989 & 1.02274 & 1.01765 & 1.01364 \\
\hline 5200 & 1.06222 & 1.06071 & 1.05841 & 1.05460 & 1.04928 & 1.04315 & 1.03580 & 1.03055 & 1.02638 \\
\hline 5400 & 1.07544 & 1.07355 & 1.07101 & 1.06704 & 1.06157 & 1.05531 & 1.04778 & 1.04239 & 1.03807 \\
\hline 5600 & 1.08761 & 1.08533 & 1.08256 & 1.07844 & 1.07284 & 1.06645 & 1.05878 & 1.05326 & 1.04881 \\
\hline 5800 & 1.09881 & 1.09616 & 1.09316 & 1.08889 & 1.08318 & 1.07668 & 1.06888 & 1.06324 & 1.05869 \\
\hline 6000 & 1.10914 & 1.10611 & 1.10288 & 1.09849 & 1.09267 & 1.08607 & 1.07817 & 1.07243 & 1.06777 \\
\hline 6200 & 1.11866 & 1.11528 & 1.11182 & 1.10730 & 1.10140 & 1.09471 & 1.08671 & 1.08089 & 1.07614 \\
\hline 6400 & 1.12745 & 1.12371 & 1.12004 & 1.11540 & 1.10942 & 1.10266 & 1.09458 & 1.08868 & 1.08384 \\
\hline 6600 & 1.13558 & 1.13149 & 1.12760 & 1.12285 & & & & & 1.09096 \\
\hline 6800 & & & & 1.12972 & 1.12360 & 1.11672 & 1.10852 & 1.10249 & 1.09752 \\
\hline 7000 & 1.15006 & 1.14529 & 1.14099 & 1.13604 & & 1.12294 & & 1.10862 & 1.10359 \\
\hline 7200 & 1.15650 & 1.15142 & 1.14692 & 1.14188 & & & & & 1.10920 \\
\hline 7400 & 1.16246 & 1.15708 & & & & & & & \\
\hline 7600 & & & & & & & & & 1.11920 \\
\hline 7800 & & & & & & & & & 1.12366 \\
\hline 8000 & 1.17791 & 1.17167 & 1.16644 & 1.16108 & 1.15471 & 1.14764 & 1.13928 & 1.13304 & 1.12780 \\
\hline
\end{tabular}

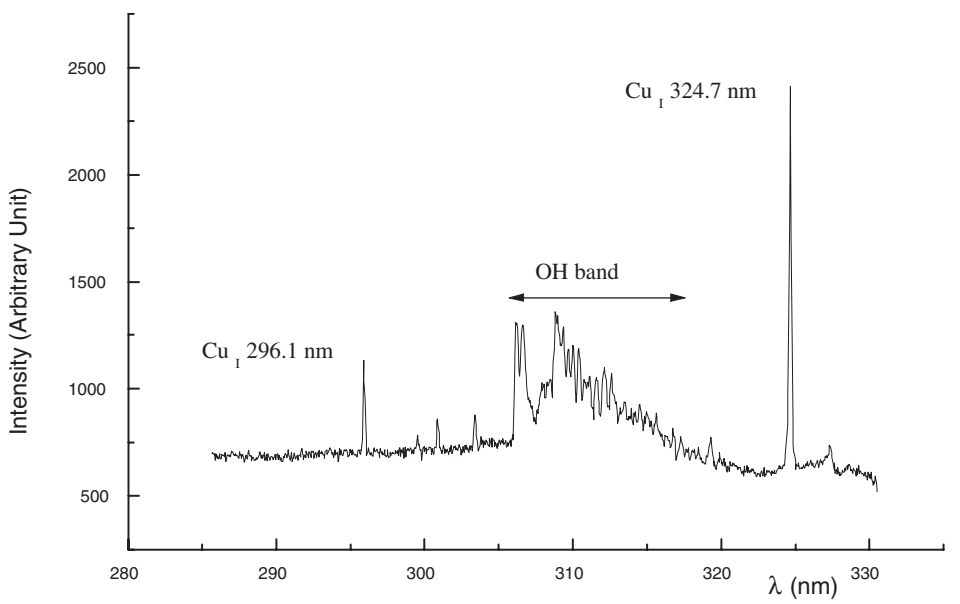

Figure 5. Spectrum recorded in the recombining plume of the laminar plasma jet. One can notice the presence of $\mathrm{Cu}_{\mathrm{I}}$ atomic lines and the $\mathrm{UV} \mathrm{OH}$ band at $306.357 \mathrm{~nm}$

Since the laminar plasma jet acts as a pump (figure 4) for the ambient air [6], we found that it was possible to observe the UV OH band at $306.357 \mathrm{~nm}$ without seeding the working gas with water vapour, because of the small amount of water vapour present in ambient air.
A typical recorded spectrum is given in figure 5, and exhibits both the presence of neutral copper lines, and of the UV OH band at $306.357 \mathrm{~nm}$. The copper lines come from the anode that is easily eroded when the plasma jet is laminar.

The determination of the temperature can be performed 


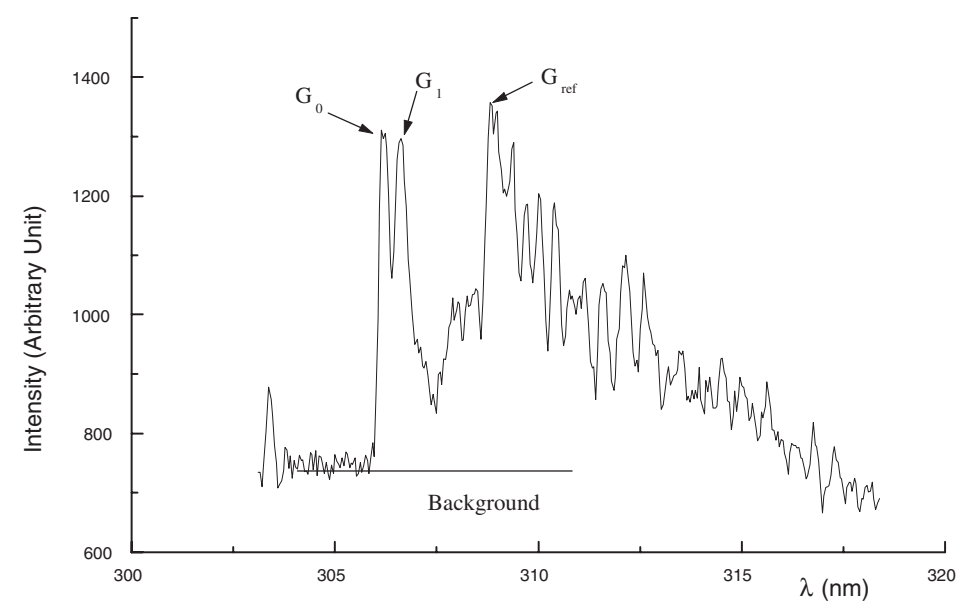

Figure 6. UV OH molecular band recorded in the plume of an argon plasma jet in ambient air.

Table 6. Atomic parameters of the copper lines observed in the plasma jet.

\begin{tabular}{llll}
\hline $\begin{array}{l}\text { Wavelength } \\
\lambda(\mathrm{nm})\end{array}$ & $\begin{array}{l}\text { Energy of the } \\
\text { upper level } \\
\left(\mathrm{cm}^{-1}\right)\end{array}$ & $\begin{array}{l}\text { Statistical } \\
\text { weight } \\
g_{u}\end{array}$ & $\begin{array}{l}\text { Transition } \\
\text { probability } \\
A\left(10^{8} \mathrm{~s}^{-1}\right)\end{array}$ \\
\hline 296.12 & 44963 & 8 & 0.0376 \\
324.75 & 30784 & 4 & 1.39 \\
\hline
\end{tabular}

using the intensity ratio of the two main $\mathrm{Cu}_{\mathrm{I}}$ lines available in the spectrum. The parameters of these two lines are summarized in table 6 [8].

The intensity ratio of the two lines can be expressed as a function of the temperature $T$ under the assumption of local thermodynamic equilibrium. Using the subscripts ' 1 ' and ' 2 ' for the two lines, we have:

$$
T=\frac{E_{2}-E_{1}}{k} \ln \left(\frac{I_{1} v_{1} g_{2} A_{2}}{I_{2} v_{2} g_{1} A_{1}}\right)
$$

where $I_{1}$ and $I_{2}$ represent the intensities, $v_{1}$ and $v_{2}$ the frequencies of the two lines, and $k$ is the Boltzmann constant. The application of the intensity ratio gives the temperature $T=4400 \mathrm{~K}$.

Figure 6 gives a plot of the UV OH band observed in the recorded spectral area. The three groups of unresolved rotational lines, labelled $G_{0}, G_{1}$ and $G_{\text {ref }}$, are easily identified (see figure 2). We have to emphasize the presence of a $\mathrm{Cu}_{\mathrm{I}}$ line located at the wavelength $\lambda=306.341 \mathrm{~nm}$ that may give a perturbation in the intensity of the group of lines $G_{0}$. For this reason, the group of lines $G_{0}$ will not be used to carry out a temperature value.

From the measured intensity ratio of the two groups of lines, we obtain a rotational temperature of $3800 \mathrm{~K}$, that is lower than the excitation temperature obtained from the intensity ratio of the copper lines. However, the rotational temperature determined by the $\mathrm{OH}$ spectrum calibration is very close to the temperature obtained with interferometry [7], that is an enthalpic temperature obtained using Gladstone's law.

\section{Conclusion}

In this paper, we have presented a very sensitive variation of a group of unresolved lines in the UV OH spectrum, that have been calibrated using numerical simulations. Calibration data are given in table form for practical use. It is clear that the main errors in a rotational temperature determination come from:

- the determination of the underlying background continuum, necessary to measure the maximum amplitudes of the groups of unresolved rotational lines;

- the evaluation of the apparatus function.

A possible direct application of this work is the elaboration of an 'OH molecular pyrometer', actually studied in our research group. A paper presenting and giving the computer program used to produce the synthetic spectra is presently in preparation.

\section{References}

[1] Gaydon A G 1957 The Spectroscopy of Flames (London: Chapman and Hall)

[2] Pellerin S, Koulidiati J, Motret O, Musiol K, De Graaf M, Pokrzwka B and Chapelle J 1997 High Temp. Mater. Process. $1493-509$

[3] Pellerin S, Cormier J M, Richard F, Musiol K and Chapelle J 1996 J. Phys. D: Appl. Phys. 29 726-39

[4] Diecke G H and Crosswhite H M 1961 J. Quant. Spectrosc. Radiat. Transfer 297

[5] de Izarra C, Vallée O and Chapelle J 1993 High Temp. Chem. Process. 2 107-13

[6] Czernichowski A 1970 Acta Phys. Pol. A 40 283-94

[7] Ranson P, Vallée O and Chapelle J 1977 Revue Phys. Appl. 121187

[8] Reader J, Corliss C H, Wiese W L and Martin G A 1980 Wavelengths and Transition Probabilities for Atoms and Atomic Ions (Washington, DC: National Bureau of Standards) 